\title{
Advances in Stroke Prevention in 2018
}

\author{
M. Edip Gurol, ${ }^{\mathrm{a}}$ Jong S. Kim ${ }^{\mathrm{b}}$ \\ aDepartment of Neurology, Massachusetts General Hospital, Harvard Medical School, Boston, MA, USA \\ ${ }^{b}$ Department of Neurology, Asan Medical Center, University of Ulsan College of Medicine, Seoul, Korea
}

Stroke remains one of the most important causes of disability and death worldwide. Despite all the advances in the acute treatment of ischemic strokes, the proportion of patients who qualify for such medical and endovascular interventions is small and the outcomes are not good even for many who undergo acute therapies. The outcomes of hemorrhagic strokes are dismal, with mortality rates ranging from $30 \%$ to $40 \%$ and exceeding 50\% for anticoagulant-related intracerebral hemorrhages. ${ }^{1}$ Extensive efforts to identify hemostatic therapies and surgical evacuation did not yield any efficient acute treatment for brain hemorrhages. Consequently, stroke prevention remains one of the most important targets in the fields of neurology, cardiology, vascular medicine, and geriatrics medicine.

Traditionally, the preventive role of the stroke neurologist consisted of identifying patients who could preferentially benefit from aspirin versus warfarin and reviewing patients with carotid disease as endarterectomy candidates. As simplistic as it may sound nowadays, the adoption of the Trial of Org 10172 in Acute Stroke Treatment (TOAST) classification system for the etiologic classification of ischemic strokes was a huge step forward in the 1990s. ${ }^{2}$ Clopidogrel was introduced and combination antiplatelet therapies were studied in ischemic stroke populations, as well as warfarin in non-atrial fibrillation cohorts, yielding a series of negative studies mainly because of the increased risk of intracranial hemorrhages.

The past decade brought a considerable amount of scientific data that helped in better understanding the etiologies of ischemic and hemorrhagic strokes. An important advance was the identification of cerebral small vessel diseases as the main cause of hemorrhagic strokes and their interaction with anticoagulants in causing fatal or highly disabling brain hemorrhages. This line of research also resulted in a large gamut of imaging markers of brain microangiopathies, such as microbleeds, superficial sidero- sis, and enlarged perivascular spaces, as well as magnetic resonance imaging measures of structural and functional cerebral integrity. ${ }^{3}$ Hemorrhagic damage related to cerebral small vessel diseases is closely tied to microangiopathic ischemic injury represented by lacunar infarcts, white matter disease, and brain atrophy. Anticoagulants and combination antithrombotics are not more beneficial than aspirin alone in a population with brain small vessel diseases; however, they increase the risk of intracerebral hemorrhage. The specific considerations for brain microangiopathies that stroke experts need to be familiar with are discussed in the first article of a series of reviews in this issue of the Journal of Stroke, "Updates on prevention of hemorrhagic and lacunar strokes."4 To our knowledge, this is the first comprehensive review that specifically addresses the issue of preventing both intracerebral hemorrhages and lacunar infarcts, seemingly opposite consequences of the same disease process: brain microangiopathies. This review also includes critical data for predicting the risk of brain bleeds before they occur, by using imaging and other markers, a major issue commonly encountered in contemporary cardioembolic stroke prevention efforts.

Several major advances marked the field of cardioembolic stroke prevention in recent years. The second article of the review series in this issue of the Journal of Stroke, "Updates on prevention of cardioembolic strokes," details these advances. ${ }^{5}$ Non-vitamin K antagonist oral anticoagulants (NOACs) performed well against suboptimally performing warfarin arms in non-valvular atrial fibrillation trials and became the first line of stroke prevention in these patients. These medications have many practical advantages over warfarin, and physicians have gained experience in their use. The recent guidelines of the European Heart Rhythm Association provide extensive data on their proper use, including cautions in commonly encountered populations such as patients with renal failure. ${ }^{6}$ Despite all 
these advances, stroke prevention strategies are underused in patients with atrial fibrillation, including those at a higher embolic risk. The major fear in prescribing warfarin and NOACs is their association with severe intracranial hemorrhages. It is hoped that the first review discussed above will provide adequate information to differentiate patients who are not at a high risk for brain bleeding from those who are. This distinction is critical as patients with atrial fibrillation at a high embolic risk but without a high hemorrhagic risk should be encouraged to use long-term warfarin or NOAC. These medications rely on daily regular use for efficacy, and identifying patients with low hemorrhage risk allows clinicians to reinforce the importance of strict adherence to anticoagulation. Patients with atrial fibrillation at a higher hemorrhage risk present a therapeutic dilemma; nevertheless, there had also been important advances on that front. The review on cardioembolic stroke includes a discussion of novel nonpharmacological methods that do not rely on lifelong anticoagulant use for stroke prevention in atrial fibrillation. Left atrial appendage closure with the WATCHMAN (Boston Scientific, Marlborough, MA, USA) device is the first and currently only method approved by the United States Food and Drug Administration for a clinical indication of stroke prevention in patients with atrial fibrillation who have a rationale to seek non-anticoagulant measures. ${ }^{1}$ This second article of the review series on stroke prevention discusses clinically relevant aspects of left atrial appendage closure procedures, including their strengths and potential complications. Another detailed discussion is on patent foramen ovale as a potential stroke etiology and the role of its closure in preventing recurrent events. Extensive information is provided on the workup and findings in younger patients with stroke, which is necessary to attribute the ischemic event to patent foramen ovale. The most clinically relevant aspects of the major studies on NOACs, left atrial appendage closure and patent foramen ovale closure procedures are provided in tables, in a hope to facilitate translation of data in individual patient care. Advances in stroke prevention in infective endocarditis, heart failure, and valvular and coronary heart diseases are also discussed.

Finally, the third article of the series, "Updates on prevention of atherosclerotic strokes," reviews emerging data on physiopathological mechanisms, diagnosis, and both medical and interventional treatments of cerebrovascular atherosclerosis. ${ }^{8}$ The relevance of these pathologies to stroke neurology practice cannot be overemphasized. Microembolic signal detection with transcranial Doppler and high-resolution magnetic resonance imaging of the cerebral vessel wall are some of the trendy diagnostic topics discussed. Novel data on the control of classical vascular risk factors, antithrombotic agents, and anti-inflam- matory treatments are presented. This article also provides specific recommendations for the management of symptomatic and asymptomatic cerebrovascular stenoses.

We hope that the focused stroke prevention series published in this issue of the Journal of Stroke will help clinicians in evaluating all aspects of concurrent ischemic and hemorrhagic risks in patients, and in providing individualized care incorporating the latest advances in the field.

\section{References}

1. Gurol ME. Nonpharmacological management of atrial fibrillation in patients at high intracranial hemorrhage risk. Stroke 2018;49:247-254.

2. Adams HP Jr, Bendixen BH, Kappelle ப, Biller J, Love BB, Gordon $\mathrm{DL}$, et al. Classification of subtype of acute ischemic stroke. Definitions for use in a multicenter clinical trial. TOAST. Trial of org 10172 in acute stroke treatment. Stroke 1993; 24:35-41.

3. Wardlaw JM, Smith C, Dichgans M. Mechanisms of sporadic cerebral small vessel disease: insights from neuroimaging. Lancet Neurol 2013;12:483-497.

4. Tsai HH, Kim JS, Jouvent $E$, Gurol ME. Updates on prevention of hemorrhagic and lacunar strokes. JStroke 2018;20:167-179.

5. Topcuoglu MA, Liu L, Kim DE, Gurol ME. Updates on prevention of cardioembolic strokes. J Stroke 2018;20:180-196.

6. Steffel J, Verhamme P, Potpara TS, Albaladejo P, Antz M, Desteghe L, et al. The 2018 European Heart Rhythm Association practical guide on the use of non-vitamin $\mathrm{K}$ antagonist oral anticoagulants in patients with atrial fibrillation. Eur Heart $J$ 2018;39:1330-1393.

7. Hsu JC, Maddox TM, Kennedy KF, Katz DF, Marzec LN, Lubitz $S A$, et al. Oral anticoagulant therapy prescription in patients with atrial fibrillation across the spectrum of stroke risk: insights from the NCDR PINNACLE registry. JAMA Cardiol 2016;1:55-62.

8. Tsivgoulis G, Safouris A, Kim DE, Alexandrov AV. Recent advances in primary and secondary prevention of atherosclerotic stroke. J Stroke 2018;20:145-166.

Correspondence: M. Edip Gurol

Department of Neurology, Massachusetts General Hospital, Hemorrhagic

Stroke Research Program, 175 Cambridge Street, Suite 300, Boston, MA 02114 USA

Tel: +1-617-726-5362

Fax: +1-506-700-2420

E-mail:edip@mail.harvard.edu

The authors have no financial conflicts of interest. 